\title{
Longitudinal Relaxation Time
}

National Cancer Institute

\section{Source}

National Cancer Institute. Longitudinal Relaxation Time. NCI Thesaurus. Code C80363.

The time it takes for the net nuclear spin in the plane that is parallel to the magnetic field of a spectrometer to return to its equilibrium value. 